\title{
Methods of constructing basis in solving inverse problems
}

\author{
A.V.Mozgovoy, V.S.Abramchuk, I.V.Abramchuk* \\ M.Kotsyubynsky Vinnitsa State Pedagogical University, \\ 32 Ostrozsky Str., 21100 Vinnitsa, Ukraine \\ "Vinnitsa National Technical University, 47 Khmelnitsky highway Ave., \\ 21103 Vinnitsa, Ukraine
}

\section{Received April 4, 2014}

\begin{abstract}
The paper suggests theoretical bases for building the complete systems of the Taylortype basic elements with dense range of derivatives of positive, negative and fractional orders. This enables to explore the qualitative behavior of the solutions to differential and integral equations (direct and inverse problems), to process the experimental data, to identify the systems, to specify mathematical models of the objects and processes, to optimize the designing projects with specified characteristics, to prognosticate natural phenomena.
\end{abstract}

Предложены теоретические основы построения полных систем базисных әлементов тейлоровского типа с плотным спектром производных целых положительных, отрицательных и дробных порядков. Это позволяет исследовать качественное поведение решений дифференциальных и интегральных уравнений (прямых и обратных задач), обрабатывать экспериментальные данные, идентифицировать системы, уточнять математические модели объектов и процессов, оптимизировать конструкторские проекты по заданным характеристикам, осуществлять прогноз природных явлений.

Методи побудови базису при розв'язуванні обернених задач. О.В.Мозговий, В.С.Абрамчук, I.В.Абрамчук. Запропоновано теоретичні основи побудови повних систем базисних елементів тейлорівського типу з щільним спектром похідних цілих додатних, від'ємних та дробових порядків. Це дозволяє досліджувати якісну поведінку розв'язків диференціальних та інтегральних рівнянь (прямих та обернених задач), обробляти експериментальні дані, ідентифікувати системи, уточнювати математичні моделі об'єктів та процесів, оптимізувати конструкторські проекти з заданими характеристиками, здійснювати прогноз природних явищ.

\section{Introduction}

Components manufacturing follows a special technological process flow diagram, which ensures the output operation capacity of the end product. One of the most significant tasks in designing assignments stipulates for the possibility to obtain the input figures for the physical and mechanical characteristics of constructive materials used in their manufacturing and their structure, considering the output factors.
The formulation of the above problem refers it to the inverse one.

An important feature of the inverse problems that arise in processing the experimental data is that the obtained information is approximate. Considering the above, the issues which are related to the existence and uniqueness in the solution of these tasks, as well as continuous dependence of the solution on the input data of the reverse tasks require its further analysis. This analysis shall be carried out within the 
specified mathematical model - the operator equation $A z=u$, where the class of elements $z \in Z$ should contain $\tilde{z}$-characteristics of the real object or a process, and class $U$, possessing the right part of the equation $u$, shall be determined by the type of experimental information. It is being assumed that the element $\tilde{u}=A \tilde{z}$, which is under the determination during the experiment, exists in the class $U[1-3]$.

Since the experiment is conducted approximately, the element $\tilde{u}$ is unknown, the element $u_{0}$ is available and the error $\delta u$, which characterizes degree of approximation $u_{0}$ to $\tilde{u}$ in some matrix, is acceptable.

Formation of the mathematical model ( $A$, $Z, U)$ stipulates for determination of such notions as excitation, stability, quality in solution, its equability [4], substantiation of rules for building basis of space $Z$, $U$, adequate to the object (process) under research, and application of methods for the approximate solution of the equation $A z=u, z \in Z$, $u \in U$ (solution of the incorrect problems [5]).

\section{Formulation of the problem}

The inverse problems have their own peculiarities [1-3]. Firstly, they are non-linear in sense that the characteristics or parameters to be determined usually occur in the operator equations nonlinearly. Secondly, solution to the majority of the inverse problems is not the unique one. The insurance of the uniqueness often requires supplementary experimental information, which enters an additional error in the solution of the problem. Thirdly, the inverse problems are incorrect since the input information in the inverse problems is usually taken with some experimental error, which may not be always estimated. For the solution of the incorrect problems there shall be used different methods of regularizations which reduce the solution of the inverse problem to the problem of determining the minimum of functional [5].

The problem of the functional minimization may be solved efficiently only by choosing the complete basis that adequately describes the object under research. The same matter arises during solution of the direct problems. Hard hyperbolic polynomials of the Taylor's type with positive integer orders derivatives have been suggested for the solution of complex practically significant problems, described by stiff differential equations and the energy dissipation problems [8, 9]. Identification of the contemporary viscoelastic materials requires the bases of the Taylor's type not only with positive integer derivatives, but with negative and fractional derivatives [6, 13].

The objective of the paper is to lay down the rules for generation of the full system of basic elements and topological assumptions as well as their applicability to the solution of the inverse problems.

\section{Research results}

Since the experiment resulted in the discrete function $u$, which is to be differentiated and integrated for solution of the direct and inverse problems of mathematical simulation, let first introduce the topological assumptions similar to topology of the Taylor series [4].

Let us set up the Taylor series of differentiated function $f(x)$ in order of point $x_{0}$ of space $R^{n}[4]$ :

$$
\begin{gathered}
f(x)=f\left(x_{0}\right)+\sum_{i}\left(x-x_{0}\right)_{i} f_{i}+ \\
+\frac{1}{2 !} \sum_{i j}\left(x-x_{0}\right)_{i}\left(x-x_{0}\right)_{j} f_{i j}+\ldots .
\end{gathered}
$$

If we restrict ourselves the equation (1) only the first members to the $k$-th degree, the coefficients $f, f_{i}, f_{i j}, \ldots, f_{i j \ldots k}$ can be regarded as elements of the space of $R^{D}$ dimension [4]

$$
\begin{gathered}
D= \\
=1+n+n \frac{(n+1)}{2 !}+\ldots+\frac{(n+k-1) !}{k !(n-1) !}=\frac{(n+k) !}{n ! k !},
\end{gathered}
$$

where $D$ - the number of the elements in the equation (1), namely: constant term 1 , linear term - $n$, squared term $n(n+1) / 2$ !, $k$-th terms - $(n+k-1) ! / k !(n-1) !$.

Topology in the space $R^{D}$ shall be determined as follows: if $f, g-$ the two differential functions, the distance between them may be set as follows [4]:

$$
\begin{array}{r}
\|f-g\|_{x_{0}}=\left|f\left(x_{0}\right)-g\left(x_{0}\right)\right| P+\sum_{i}\left|f_{i}-g_{i}\right|^{P}+(3) \\
\sum_{i j}\left|f_{i j}-g_{i j}\right| P+\ldots+\sum_{i j \ldots k}\left|f_{i j \ldots k}-g_{i j \ldots k}\right| P, \quad 1 \leq P<\infty .
\end{array}
$$

Open sets as well as topology may be determined differently depending on the way how the distance had been set between functions, including in the $\varepsilon$-order of the function $f$ all the functions $g$, which are located from the function $f$ on distance which is less than $\varepsilon$. Thus the attribution 
(2) generates the equivalent $C^{k}$ topologies in the point $x_{0}$ (if $k \rightarrow \infty$, we get the topology $C^{\infty}$ in the point $x_{0}$ ).

Formation of the topology, determined by the coefficients of the Taylor series, calculated to the $k$-th degree requires the functions $f(x)$ to be the $k$-times differentiated (and it is sufficient for it to be analytical in the point $x_{0}$ ) [4]. The similar problem does not appear when we talk about application of the topology (2) to the solution of the problems in which the solution shall be described by analytical functions. During the solution of the inverse problems the input information is the discrete functions only. Therefore the direct application of the Taylor series to the solution of the inverse problems appears to be impossible.

Since the qualitative theory of the potential and autonomous dynamic systems is based on the topology of the Taylor series which may not be directly transferred on the inverse problems, it is necessary to create the basis for formation of the full systems of basic elements which could adhere the principal peculiarities of the Taylor series topology and at the same time would not require the analytic property of the data of investigating mathematical model $A z=u, z \in Z, u \in U$.

The germ of the Taylor type polynomial shall be the analytical function $P(x), x \in \Omega \subset$ $R$, derivatives from which shall form a linearly independent system. Thus, the class $K$ of the functions which create a basis over the field of real numbers shall be determined as: $\forall n \in N$ determine $P_{n}(x)=P_{n-1}^{\prime}(x), \quad P_{1} x=$ $P_{0}{ }^{\prime}(x), P_{0}(x)=P(x), x \in \Omega \subset R$.

The generated bases have the following significant peculiarities: the basis elements do not need to be saved since they are generated recurrently following one and the same rule $P_{n}(x)=P_{n-1}(x), n \in N$; changing the germs $P(x)$ allows to choose a basis which is the most simple and the most appropriate to the real process. A basis on the ground of the Taylor-type polynomial germs may be used for approximate solution of various practical problems, since the base elements, which are determined as derivatives of the positive integer orders may be extended to the derivatives of the negative orders: $P_{-1} x=\int P_{0}(x) d x, \ldots$, $P_{-(n+1)}(x)=\int P_{-n}(x) d x$ and fractional derivatives which are determined by the RiemannLiouville or Caputo formulas [6]. The exam- ples of such basis functions which had been used for solution of the inner energy dissipation problems as well as for solution of the stiff problems are the hyperbolic polynomials which are built as germ of the functions $P(x)=\operatorname{th} x, \quad P(x)=\operatorname{ch}^{-1}(x), \quad \forall n \in$ $N: P_{n}(x)=P_{n-1}{ }^{\prime}(x)$ [7-9]. It is necessary to note that degree basis $\left\{x^{n}\right\}, n \in N$ shall be based in the germ $P(x)=1$ following the rule $\forall n \in N: P_{-n}(x)=\int P_{-(n-1)}(x) d x$.

If class $K$ of the functions is chosen, it is necessary to set the rate $\|f-g\|$, which reflects the peculiarities of the problem which is being solved, and the ultimate goal of an approximation [10-12]. Among the approximation problems it is necessary to emphasize the problems on experimental data smoothing, numerical differentiation and functions' integration, set on the grid [12]. Problem on experimental data smoothing, set on a dense grid by its values that are found with errors, provided that the replacement function should be smooth to a certain order, may be solved in the class of the Taylor-type basic elements by building an element of the best approximation in the space with the norm

$$
\|\Delta\|_{p}=\left(\sum_{x_{i} \in \Omega} \mid \Delta\left(x_{i}\right)^{p}\right)^{1 / p},
$$

where $\Omega=\left\{\begin{array}{c}\left\{x_{i}\right\} \\ m\end{array}\right.$ grid, $1 \leq p<\infty, \Delta\left(x_{i}\right)=$ $Q_{m}\left(x_{i}\right)-f_{i}=\sum_{j=1}^{m} \alpha_{j} P_{j}\left(x_{i}\right)-f_{i},\left\{f_{i}\right\}^{m}{ }_{i=1}-\operatorname{grid}$ function, $Q_{m}(x)-$ polynomial, $\left\{P_{j}(x)\right\}^{m}{ }_{j=1}$ - elements of the base functions of the Taylor-type polynomial.

Polynomial (element) of the best approximation $Q_{m}{ }^{*}(x)$ shall be found on the assumption of minimizing the norm $\|\Delta\|_{p}$ on the vector of parameters $\alpha=\left(\alpha_{1}, \ldots, \alpha_{m}\right)^{T}$ and must satisfy the conditions: there is only polynomial $Q_{m}{ }^{*}(x)$, for which the upper bounds $\|\Delta\|_{p}$ and optimal parameters $\alpha_{i}$ of the approximation is easy to find [10-12].

Thus during the solution of the inverse problems there appears the main task, which may be formulated as follows: it is necessary to approximate the function $f$, which describes the real process, set in the points from $\Omega$ with an error - that is, instead of $f(x)$ we get $f(x)$ and the upper bound is known $\|f-f\|$. Let's consider $Q^{*}(f)$, $Q^{*}(\mathcal{f})$ - polynomials of the best approximation. How $Q^{*}\left(\mathcal{f}^{\prime}\right)$ differs from the polynomial 
$Q^{*}(f)$ and is it possible to make the value $\left\|Q^{*}(f)-Q^{*}\left(f^{\prime}\right)\right\|$ arbitrarily small if $\|f-f\|$ shall be reduced? A positive answer to these questions follows from the theorems [10, p.80; 11, p.100].

Germs of the Taylor-type polynomials for the functions $P(x, y, \ldots, t)$ of many variables shall be built on the analogy with the functions of one variable, replacing the ordinary derivatives by partial. It is often convenient to specify the function $P$ as a product $P=P_{1}(\mathrm{x}) P_{2}(y) \ldots P_{k}(t)$. The Taylortype polynomials are easy to reduce to the orthogonal ones on the base of the GramSchmidt orthogonalization in spaces with the set scalar product [9].

\section{Applicability of the Taylor-type base to solution of the inverse problems}

\subsection{Coefficient inverse problems.}

Set of coefficient inverse problems in Mechanics of Solids is a rapidly developing section in Experimental Mechanics, and requires a thorough theoretical substantiation [3]. Let's consider the problem on identification of visco-elastic materials, which are able to absorb (remember) the part of energy spent on their deformation, and dissipate the rest of it. There is a range of the basic models in continuum Mechanics, describing the relationships between the strength and deformations which form the basis for the view of the general differential equation of the visco-elastic system (operator equation of the mechanical system $[13,14]$ )

$$
\begin{aligned}
& {\left[1+\sum_{k=1}^{p} a_{k} \frac{d^{\beta_{k}}}{d t^{\beta_{k}}}\right] \sigma(t)=} \\
= & {\left[m+\sum \sum_{k=1}^{q} b_{k} \frac{d \beta_{k}}{d t^{\beta_{k}}}\right] \varepsilon(t), }
\end{aligned}
$$

where $\beta_{k}=k+\beta-1, \quad 0<\beta \leq 1, d^{\beta_{k}} / d t^{\beta_{k}}-$ fractional derivatives, which, when $\beta=1$ are the usual derivatives, $\sigma(t)-$ tension applied to the body, $\varepsilon(t)-$ deformation. From the law (4) there follow the basic versions $(p=q=1)$ of the Hook's model $(a=b=0)$, Newton $(a=m=0)$, Voigt ( $a=0)$, Maxwell $(m=0)$ and Ziner $(k=1, \beta=1)[6,13,14]$. Let the Ziner model be chosen as the structural one [15], which adequately describes the creep, relaxation, aftereffect and has the form of $[3,15]$

$$
\tau \sigma+\sigma=E \tau \varepsilon+H \varepsilon,
$$

where $E$ - instant module, $H$ - long-term module, $\tau$ - relaxation time, and $E>H$. The inverse problem is to determine the parameters $E, H, \tau$ based on the results of the experiment, where there shall be set, for example, $\sigma(t)$ and $\varepsilon(t)$ shall be measured, from the uniaxial tensile experiment for the fixed discrete set of time points $t_{k}$ [3]. We note that experimentally taken discrete functions $\varepsilon(t), \sigma(t)$ are approximate and they must be differentiated, that is, the problem of identification is unstable (incorrect) [3].

If in the space $C^{2}(\Omega)$ there are functions $\varepsilon(t), \sigma(t)$ in which the Jacobi determinant $\Delta\left(t_{1}, t_{2}, t_{3}\right) \neq 0$, the parameters $E, H, \tau$ may be determined uniquely [1,3]. Putting $\varepsilon(t)$ $=\operatorname{th} t, \quad \sigma(t)=\operatorname{ch}^{-1}(t)$ and choosing $t_{1}=$ $0<t_{2}<t_{3}$, we obtain that the determinant $\Delta \neq$ 0 . Thus, the parameters $E, H, \tau$ may be found uniquely. Choosing functions $P(t)=$ $\mathrm{ch}^{-1} t$ and tht that, as the germs of the Taylor-type polynomial, we obtain complete sets of the basis functions in spaces of which it is possible to search for the best approximation of the discrete grid functions $\left\{\varepsilon\left(t_{i}\right)\right\}_{i=1}^{m},\left\{\sigma\left(t_{i}\right)\right\}_{i=1}^{m}$ by the methods [3, 5 , $10]$, to identify and specify the models (4), (5) for real visco-elastic materials.

\section{2 Dynamical systems stability}

During study of the mathematical models $A z=u, z \in Z, u \in U$ of the inverse problems, we theoretically substantiate the question of continuous dependence of the solution on the right-hand side perturbation [13]. These studies however do not explain the physical content of stability of dynamic systems up to the variations of their parameters, as the problem is quite complex. To eliminate this gap it is necessary to use the conclusions of the catastrophe theory [4] in optimization algorithms for solving the direct and inverse problems.

Let's the dynamic system be described by the system of differential equations $[4,16]$

$$
\begin{gathered}
\frac{d x_{i}}{d t}=F(x, c) ; \quad i=1, \ldots, m ; \\
x=\left(x_{1}, \ldots, x_{m}\right) ; \quad c=\left(c_{1}, \ldots, c_{k}\right),
\end{gathered}
$$

where $x$ - vector of phase variables (coordinates), $c$ - vector of parameters control. 

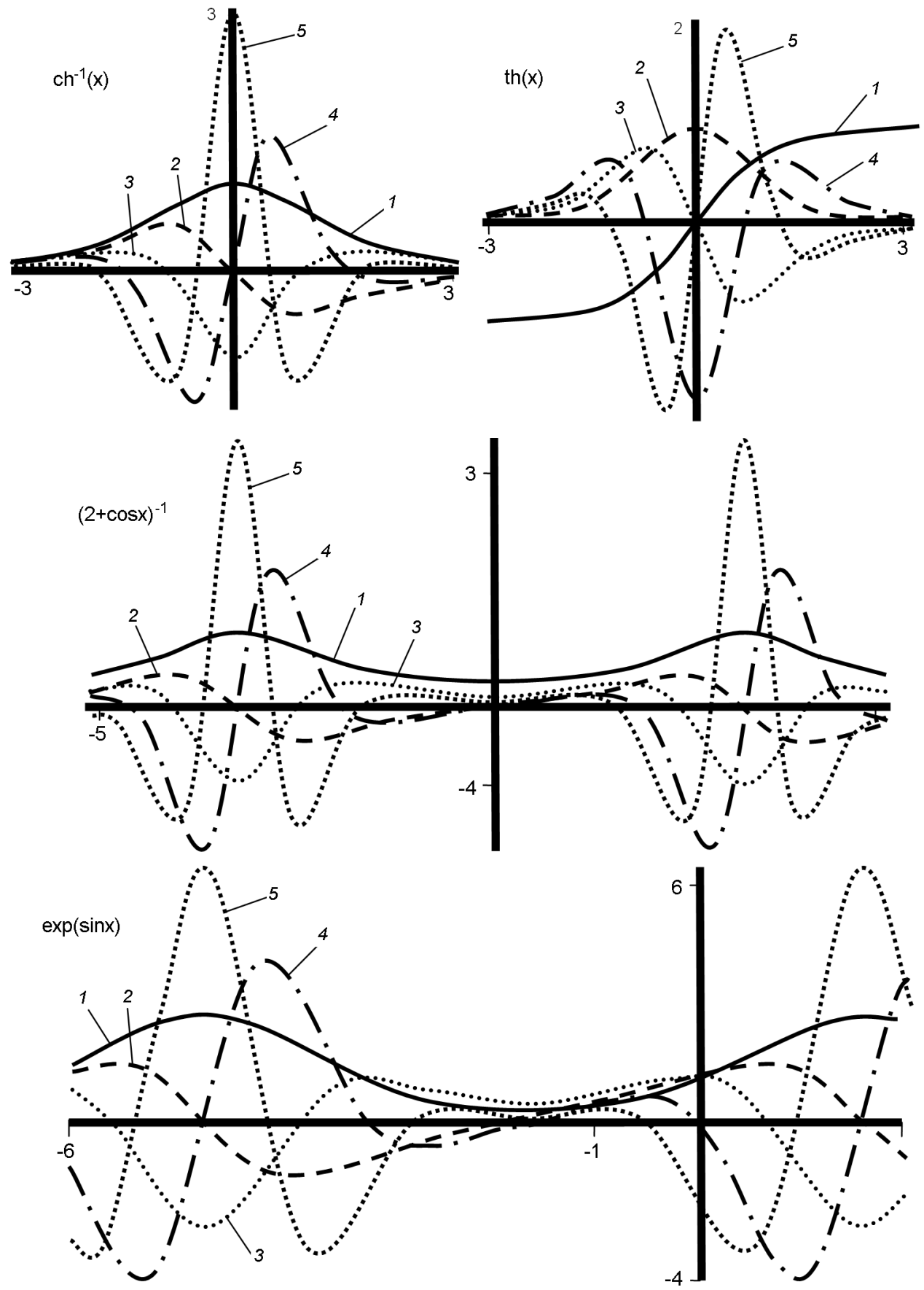

Fig. 1. Basic elements to be generated, respectively, by functions: $\operatorname{ch}^{-1} x, \operatorname{th} x,(2+\cos x)^{-1}, e^{\sin x}$. Number of the curve corresponds to the basic functions of order $n$.

The local dynamic and structural properties shall be determined by the own values of the matrix stability $F_{i j}=\partial F_{i} / \partial x_{j}$, calculated at the critical point $x_{0}$ for the set value of parameter vector $c_{0}$ [4]. Variations $\delta c_{i}$ of the parameters $c_{i}, i \in[1: k]$ at the point $c_{0}=\left(c_{1}^{0}, \ldots, c_{k}^{0}\right)$ lead to excitation in the solution of the system (6). The solution will remain structurally stable only under condition that the own values of the matrix $F_{i j}$ have neither small nor multiple own values $[4,16]$. Such researches in changing the solution to variations of the parameters may be done only in the space of the Taylor-type basic elements.

Here are some examples of germs of the Taylor-type polynomials that describe behavior of the different processes (Table, 
Table. Taylor's type basic elements: $P_{n}^{\prime}(x)=P_{n+1}(x), n=1 \ldots 6$

\begin{tabular}{|c|c|c|c|c|}
\hline$n=1$ & $P_{1}=\operatorname{ch}^{-1} x$ & $P_{1}=\operatorname{th} x$ & $P_{1}=(2+\cos x)^{-1}$ & $P_{1}=e^{\sin x}$ \\
\hline$n=2$ & $-\operatorname{ch}^{-1} x \cdot \operatorname{th} x$ & $\operatorname{ch}^{-2} x$ & $\sin x(2+\cos x)^{-2}$ & $e^{\sin x} \cdot \cos x$ \\
\hline$n=3$ & $-\operatorname{ch}^{-1} x \cdot\left(1-2 \operatorname{th}^{2} x\right.$ & $-2 \operatorname{ch}^{-3} x \cdot \operatorname{sh} x$ & $\begin{array}{c}(2+2 \cos x- \\
\left.-\cos ^{2} x\right) \cdot(2+\cos x)^{-3}\end{array}$ & $e^{\sin x} \cdot\left(\cos ^{2} x-\sin x\right)$ \\
\hline$n=4$ & $\operatorname{ch}^{-1} x \cdot \operatorname{th} x \cdot\left(5-6 \operatorname{th}^{2} x\right)$ & $2 \operatorname{ch}^{-4} x \cdot\left(2 \operatorname{ch}^{2} x-3\right)$ & $\begin{array}{c}(2+8 \cos x- \\
\left.-\cos ^{2} x\right) \cdot \sin x \cdot(2+\cos x)^{-4}\end{array}$ & $\begin{array}{c}-\cos x \cdot e^{\sin x} \cdot(1+3 \sin x- \\
\left.-\cos ^{2} x\right)\end{array}$ \\
\hline$n=5$ & $\begin{array}{l}\operatorname{ch}^{-1} x \cdot\left(5-28 \operatorname{th}^{2} x+\right. \\
\left.+24 \operatorname{th}^{4} x\right)\end{array}$ & $-8 \operatorname{ch}^{-5} x \cdot \operatorname{sh} x \cdot\left(\operatorname{ch}^{2} x-3\right)$ & $\begin{array}{c}\left(-8+32 \cos x+24 \cos ^{2} x-\right. \\
-22 \cos ^{3} x+ \\
\left.+\cos ^{4} x\right) \cdot(2+\cos x)^{-5}\end{array}$ & $\begin{array}{c}\left(3+\sin x-7 \cos ^{2} x-\right. \\
-6 \cos ^{2} x \cdot \sin x+ \\
\left.\quad+\cos ^{4} x\right) \cdot e^{\sin x}\end{array}$ \\
\hline$n=6$ & $\begin{array}{c}-\operatorname{ch}^{-1} x \cdot \operatorname{th} x \cdot\left(3-46 \operatorname{th}^{2} x+\right. \\
\left.+80 \operatorname{th}^{4} x\right)\end{array}$ & $8 \operatorname{ch}^{-6} x \cdot\left(2 \operatorname{ch}^{4} x-15 \operatorname{sh}^{2} x\right)$ & $\begin{array}{c}(-104+32 \cos x+ \\
+204 \cos ^{2} x-52 \cos ^{3} x+ \\
\left.+\cos ^{4} x\right) \cdot \sin x \cdot(2+\cos x)^{-5}\end{array}$ & $\begin{array}{c}\left(16-15 \sin x-25 \cos ^{2} x-\right. \\
-10 \cos ^{2} x \cdot \sin x+ \\
\left.+\cos ^{4} x\right) \cdot \cos x \cdot e^{\sin x}\end{array}$ \\
\hline
\end{tabular}

Fig.1 ): transitions (excitations of any nature on the finite interval $\left[0 ; t_{0}\right]$ with attenuation (in the interval $\left[t_{0}, \infty\right]$ are describing by the basis functions of the Taylor-type polynomial constructed from the germs $e^{-P n(x)}, P_{n}(x)>0-$ no high-order polynomials (e.g., $P_{2}(x)=x^{2}$ ), rational expressions $f(x) / g(x), g(x)>0, f$ and $g-$ differentiated functions (e.g., $1 /\left(1+x^{2}\right)$, $\left(e^{x}-e^{-x}\right) /\left(e^{x}+e^{-x}\right)=\operatorname{th} x, 2 /\left(e^{x}+e^{-x}\right)=\operatorname{ch}^{-1} x$, $x /\left(1+x^{2}\right)$; weakly pulsing and periodic processes are described by the germs $e^{T n(x)}$, where $T_{n}(x)$ - trigonometric polynomial (e.g., $\sin \omega x)$; processes that can predict the destruction of the dynamic system of deformation, natural disasters are described by the germs $\left(P_{n}(x)\right)^{s}, P_{n}(x)-$ not high orders polynomials, $s$ - fractional rate (for example, $\left.(1+x)^{3 / 2}\right)$. Multivariate germs of the Taylor-type polynomial are constructed on the base of one-dimensional, such as $e^{-a x^{2}-b y^{2}}$, $e^{\sin \alpha x+\cos \beta y}$ etc.

\section{Conclusions}

Solution of the inverse problems of identifications, designing and prognostication based on approximate experimental data requires the creation of the complete bases systems with dense range of derivatives of the whole positive, negative and fractional orders.

\section{References}

1. A.M.Denisov, Introduction to the Theory of Inverse Problems, Moscow State University, Moscow (1994) [in Russian].

2. V.G.Romanov, Inverse Problems of Mathematical Physics, Nauka, Moscow (1984) [in Russian].
3. A.O.Vatulyan, Inverse Problems in Solid Mechanics, Fizmatlit, Moscow (2007) [in Russian].

4. R.Gilmore, Catastrophe Theory for Scientists and Engineers, John Wiley \& Sone, New York (1981).

5. A.N.Tikhonov, A.V.Goncharsky, V.V.Stepanov, Numerical Methods for Solving Illposed Problems, Nauka, Moscow (1990) [in Russian].

6. V.V.Vasiliev, L.A.Simak, Fractional Calculus and Approximation Methods for Modeling Dynamic Systems. Scientific Publication, Ukraine National Academy of Sciences, Kiev (2008) [in Russian].

7. V.S.Abramchuk, I.V.Abramchuk, in: Problems of Computer Science and Computer Engineering (Pictet 2014); in: Proc. of the III-th Intern. Scie. Conf., Chernivtsi, Ukraine (2014), p.52.

8. A.V.Mozgovoy, V.S.Abramchuk, I.V.Abramchuk, Functional Materials, 11, 410 (2004).

9. V.S.Abramchuk, I.V.Abramchuk, Mathematical and Computer Modeling. Series: Physics and Mathematics, , 7 (2012).

10. N.Akhiezer, Lectures on the Theory of Approximations, Nauka, Moscow (1965) [in Russian].

11. A.L.Garkavi, in: Results of Science. Mathematical Analysis, VINITI, Moscow (1969), p.75.

12. V.I.Berdyshev, Yu.N.Subottin, Numerical Methods for Approximating Functions, Middle Ural Book Publishers, Sverdlovsk (1979) [in Russian].

13. F.Mainardi, Fractals and Fractional Calculus in Continuum Mechanics, Springer-Verlad (1977).

14. B.I.West, M.Bologna, P.Grigolini, Physics of Fractal Operators, Springer-Verlad (2003).

15. D.Mase, Theory and Problems of Continuum Mechanics, Mograw-Hill Book Company, New York (1970).

16. V.S.Abramchuk, I.V.Abramchuk, A.V.Mozgovoy, News Tula State University, Science, 3, 103 (2013). 